\title{
A stabilizing influence for K-fibres
}

Kinetochore fibres (K-fibres) are microtubule bundles that join kinetochores to the spindle poles. They are essential for the correct segregation of chromosomes and, as such, have been widely studied. However, although much is known about their plus-end dynamics, the same is not true of their minus-end dynamics. This study identifies one mechanism behind the stabilization of K-fibre minus ends and shows that this is required for functional spindle formation.

Acentrosomal formation of spindle microtubules makes an important contribution to spindle formation and requires the activation of spindle assembly factors (SAFs) on chromatin, which depends on a local RAN.GTP gradient. It is thought that nucleation of these microtubules in the vicinity of chromatin facilitates their capture by kinetochores, aiding efficient chromosome orientation. The minus ends are then focused at the spindle poles, and the K-fibres form.

From a search of the published literature, Meunier and Vernos identified microspherule 1 (MCRS1) as a potential RAN.GTP-dependent SAF. They showed that this protein localized specifically to chromosome-dependent microtubules and not to centrosomal asters. In metaphase, MCRS1 localized to the minus ends of K-fibre microtubules, which led them to investigate possible roles for MCRS1 in K-fibre assembly.

Silencing MCRS1 using short interfering RNAs resulted in cells that generate fewer chromosomal microtubules and arrest in mitosis. Spindles that persisted under these conditions were very unstable, underwent frequent collapse and reassembly, and were shorter. Overexpression of MCRS1 also led to defects in cell division, producing longer spindles. In addition, K-fibres were more sensitive to depolymerization triggered by cold exposure when MCRS1 was depleted. Therefore, the authors concluded that MCRS1 is involved in stabilizing K-fibres and maintaining their length, which has knock-on effects on spindle stability.

Consistently, MCRS1 localized specifically to RAN.GTP-induced microtubule asters in Xenopus laevis egg extracts. The authors showed that MCRS1 associated with importin- $\beta$ and the microtubule depolymerase mitotic centromereassociated kinesin (MCAK; also known as KIF2C). In vitro, MCRS1 prevented the MCAK-induced reduction in the average length of microtubules owing to the higher affinity of MCAK for microtubules and thus its displacement of MCAK. Moreover, silencing MCAK rescued the assembly of chromosomal microtubules in cells that lack MCRS1, confirming that MCRS1 acts to prevent MCAK-mediated depolymerization of microtubules, thus stabilizing K-fibres and spindle assembly.

So, MCRS1 is necessary for chromosomal microtubule and K-fibre minus-end stability in a RAN.GTP-regulated pathway that involves the inhibition of MCAK activity. Further work is needed to complete our understanding of this process and to elucidate how it is coordinated with other mechanisms that might also control microtubule minus-end stability.

Antony F. Bickenson

ORIGINAL RESEARCH PAPER Meunier, S. \&

Vernos, I. K-fibre minus ends are stabilized by a RanGTP-dependent mechanism essential for functional spindle assembly. Nature Cell Biol. 13 Nov 2011 (doi:10.1038/ncb2372) 